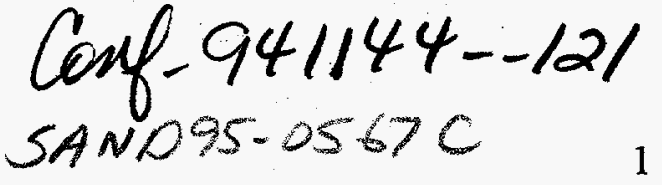

\title{
MECHANISM(S) FOR THE SUPPRESSION OF THE SWITCHABLE POLARIZATION IN PZT AND BaTiO 3
}

William L. Warren, Duane Dimos, Bruce A. Tuttle, Gordon E. Pike, Mark V. Raymond, Robert D. Nasby, R. Ramesh ${ }^{*}$ and Joseph T. Evans, Jr. ${ }^{+}$

Sandia National Laboratories, Albuquerque, NM 87185-1349

*Bellcore, Red Bank, NJ 07701

+Radiant Technologies Inc., 1009 Bradbury Ave., Albuquerque, NM 87106

\section{ABSTRACT}

Switchable polarization can be significantly suppressed in ferroelectric (FE) materials by optical, thermal, and electrical processes. The thermal process can occur by either annealing the $\mathrm{FE}$ in a reducing environment or by heating it in air to $100^{\circ} \mathrm{C}$ while impressing a bias near the switching threshold. The optical process occurs while biasing the FE near the switching threshold and illuminating with bandgap light. And the electrical suppression effect occurs by subjecting the FE to repeated polarization reversals. Using electron paramagnetic resonance, polarization-voltage measurements, and charge injection scenarios, we have been able to elucidate both electronic and ionic trapping effects that lead to a suppression in the amount of switchable polarization in FE materials. The relative roles of electronic and ionic effects in the same material can depend on the stress condition. For instance, in oxidized $\mathrm{BaTiO}_{3}$ crystals, optical and thermal suppressions occur by electronic domain pinning; electrical fatigue in the $\mathrm{BaTiO}_{3}$ crystals also appears to involve electronic charge trapping, however, it is suggested that these electronic traps are further stabilized by nearby ionic defects. In sol-gel PZT thin films with either $\mathrm{Pt}, \mathrm{RuO}_{2}$, or $\mathrm{La}-\mathrm{Sr}-\mathrm{Co}-\mathrm{O}$ electrodes it appears that the polarization suppression induced by electrical fatigue, a temperature/bias combination, or a light/bias combination are all primarily due to the trapping of electronic charge carriers to first order.

\section{INTRODUCTION}

Ferroelectric (FE) thin films are being extensively investigated for use in nonvolatile memory applications [1-4]. Since the readout process in these applications can switch the polarization state of the FE, it is important to understand any possible degradation processes that may occur over many write/read pulses, or polarization reversals. Indeed, the loss of switchable polarization $\left(\mathrm{P}^{*}\right)$ by repeated polarization reversal, a process commonly termed electrical fatigue, has been a major concern in the FE community for several decades [5-16].

Many models have been proposed to explain polarization suppression in FE materials. Some models include: electronic charge trapping at domain walls [11,14], charged ionic defect sites such as oxygen vacancies $\left(V_{0}\right)$ pinning domain walls $[3,7,12,13]$, as well as extended defect sites pinning domain walls [16]. It is impossible to distinguish between these effects from purely electrical measurements since they globally lead to a suppression in the amount of switched polarization. In order to distinguish between these various mechanisms we have used electrical, magnetic, and optical techniques to explore the cause(s) of polarization suppression induced by optical, thermal, and electrical processes in this study. We show that 


\section{DISCLAIMER}

Portions of this document may be illegible in electronic image products. Images are produced from the best available original document. 
both electronic and ionic trapping effects can in principle lead to a suppressed polarization state in FE materials. In this paper we compile and review our data on bulk single crystal $\mathrm{BaTiO}_{3}(\mathrm{BT})$ and thin film $\mathrm{Pb}(\mathrm{Zr}, \mathrm{Ti}) \mathrm{O}_{3}(\mathrm{PZT})$ showing that the relative roles of the ionic and electronic components depend on the material investigated and the particular stress condition employed [11,17-22].

\section{EXPERIMENTAL DETAILS}

The $\mathrm{BaTiO}_{3}$ single crystal plates used in this study were grown by the Remeika [23] flux method. Semi-transparent indium tin oxide (ITO) electrodes were sputter deposited on the plates for electrical contact. Polarization-voltage $(\mathrm{P}-\mathrm{V})$ measurements were made with a Radiant Technologies RT-66A tester; the applied field was along the [001] direction for the $\mathrm{BaTiO}_{3}$ samples. A $100 \mathrm{~W}$ Oriel $\mathrm{Hg}$ arc lamp was used in combination with a $3.1 \mathrm{eV}$ narrow band $(10 \mathrm{~nm})$ interference filter to optically illuminate the samples. Broad-band UV excitation was also used for some experiments to increase the total intensity and, thus, decrease the response time. Room temperature fatigue measurements on the $\mathrm{BaTiO}_{3}$ samples were induced by sinusoidal voltage cycling at $20 \mathrm{~Hz}$. The EPR measurements were only performed on the $\mathrm{BaTiO}_{3}$ crystals. The measurements were made at $20 \mathrm{~K}$ using a Bruker ESP-300E X-band spectrometer. At this temperature the $\mathrm{BaTiO}_{3}$ exhibits rhombohedral symmetry. Since the EPR lines in these crystals are very narrow, the relative error is estimated to be $5 \%$.

Substrates with bottom electrodes for PZT thin film capacitors were prepared by depositing or thermally growing $600 \mathrm{~nm}$ of $\mathrm{SiO}_{2}$ onto a (100) Si wafer, sputtering $50 \mathrm{~nm}$ of Ti as an adhesion layer, and sputtering $200 \mathrm{~nm}$ of $\mathrm{Pt}$ or $100 \mathrm{~nm}$ of $\mathrm{RuO}_{2}$. PZT 30/70, 40/60, and $53 / 47$ thin films were fabricated from $0.4 \mathrm{M}$ alkoxide derived solutions that were prepared from lead (IV) acetate, zirconium tetrabutoxide-butanol, and titanium tetraisopropoxide precursors. Further PZT processing details can be found elsewhere [11,17-19]. The top electrode consisted of a sputter deposited array of $10 \mathrm{~nm}$ semi-transparent $\mathrm{Pt}$ or $\mathrm{RuO}_{2}$ dots. Room temperature electrical fatigue was induced by subjecting PZT capacitors to sinusoidal voltage cycling.

\section{POLARIZATION SUPPRESSION IN BULK SINGLE CRYSTAL BaTiO 3}

\section{A. OPTICALLY INDUCED SUPPRESSION}

We begin by illustrating optically-induced suppression in $\mathrm{BaTiO}_{3}$ crystals. Figure 1 shows the hysteresis measurements of $\mathrm{BaTiO}_{3}$ crystals exposed to various UV-light/applied bias combinations. Figure 1 (a) shows the initial hysteresis loop of the $\mathrm{BaTiO}_{3}$ crystal. The hysteresis loop shown in Fig. 1 (b) was taken after first poling the $\mathrm{BaTiO}_{3}$ crystal to the positive remanent polarization $\left(P_{r}\right)$ and then illuminating with broad-band UV light under a bias of $-6 \mathrm{~V}$. In this case, the illuminated electrode was biased negatively with respect to the grounded bottom electrode. The switchable polarization, has been suppressed by almost $90 \%$ by this UV/bias treatment. The photo-induced suppression of the hysteresis loop is reproducible, and, being consistent with the PLZT nomenclature will be described as optically written [24]. The optically written state can also be achieved by a positive bias/UV treatment (the positive bias is applied after poling the capacitor to - $\mathbf{P}_{\mathbf{r}}$ ). In either case, we 
find that the amount the P-V loop is suppressed strongly depends on the bias voltage and is maximized by a bias below the dark coercive voltage. This phenomenon will be discussed at great length later.

Figure 1: P-V measurements of $\mathrm{BaTiO}_{3}$ crystals subjected to various UV/bias treatments: (a) initial, (b) -6V/UV combination for $10 \mathrm{~min}$.(optically written), and (c) subsequent $+25 \mathrm{~V} / \mathrm{UV}$ process for $10 \mathrm{~min}$. (optically erased).

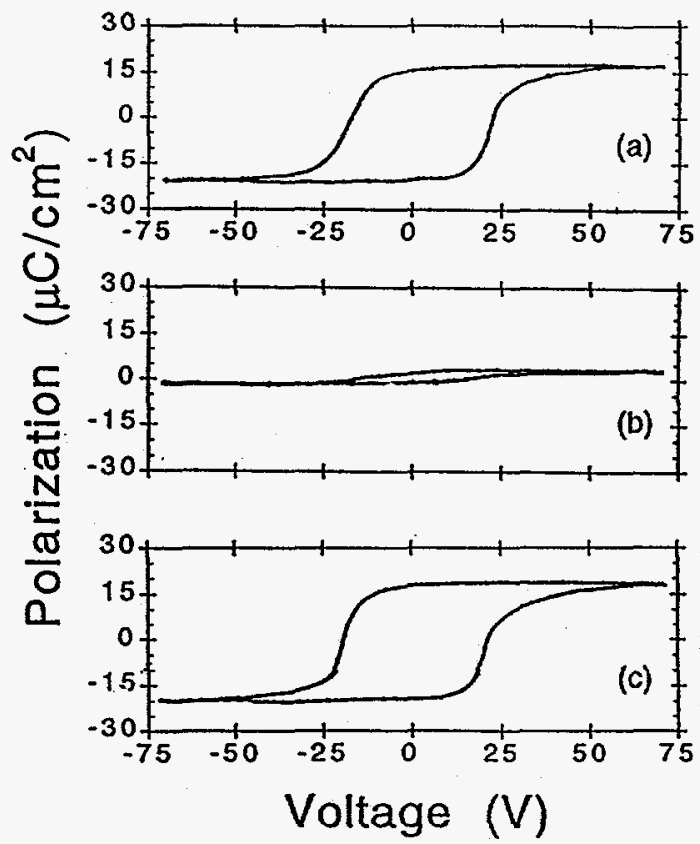

The written state can be optically erased by illuminating the crystal with UV light while applying the opposite saturating bias (a bias corresponding to the saturation voltage) to which the sample was originally written. Figure 1 (c) illustrates the particular case in which the sample was erased by a $+25 \mathrm{~V} / \mathrm{UV}$-light combination. The crystals can be repeatedly written and erased via this bias/UV approach.

Dimos et al. [17-19] have proposed a model in which the photo-induced hysteresis changes can be explained by considering the interaction between differing domain configurations and the photo-generated carriers. The essence of the model is that the photo-generated carriers become trapped at domain boundaries that have a polarization discontinuity, i.e., 900 boundaries, head-to-head, or tail-to-tail configurations. In the case of head-to-head or tail-totail configurations, the trapped charge can now enable these energetically unfavorable configurations to exist. Domain reorientation is no longer possible since the trapped charge has inhibited their motion, thereby reducing the amount of switchable polarization. The suppressed P-V loop can be restored if the trapped carriers recombine with carriers of the opposite sign; this allows the previously locked domains to reorient. Consistent with this picture, we note that optical erasure in bulk samples (thickness $>>$ optical absorption depth) can only occur if the opposite bias is applied to which the sample was originally written, i.e., propagation of the opposite carrier type in the material.

Next, EPR measurements were employed in concert with the P-V measurements to investigate the possible atomistic origin of the optically-induced polarization suppression. The paramagnetic impurity-related defect centers observed were the isolated $\mathrm{Fe}^{3+}$ and $\mathrm{Pt}^{3+}$ 
centers and $\mathrm{Fe}^{3+}-\mathrm{V}_{0}$ complexes. The $\mathrm{Fe}^{3+}-\mathrm{V}_{0}$ complex is simply an $\mathrm{Fe}$ center intimately associated to an oxygen vacancy; whereas, the isolated $\mathrm{Fe}^{3+}$ is not associated with any other particular defect site. Both $\mathrm{Fe}$ and $\mathrm{Pt}$ impurities have been effectively demonstrated to replace the $\mathrm{Ti}$ ion in the octahedron [25-28]. The combined P-V and EPR results are plotted in Fig. 2 for various room temperature illumination/bias treatments. In Fig. 2, the written state corresponds to a $-6 \mathrm{~V} / \mathrm{UV}$ combination (propagation of electrons in the material); whereas, the erased state corresponds to a $+25 \mathrm{~V} / \mathrm{UV}$ combination (propagation of holes in the material). The switchable polarization can be repeatedly suppressed and restored via this bias/UV combination. The EPR measurements indicate that the isolated $\mathrm{Fe}^{3+}$ signal intensity follows a trend similar to the switchable polarization. We note that $\mathrm{Fe}^{4+}$ ions in $\mathrm{BaTiO}_{3}$ cannot be sensed via EPR. No trends were observed for the isolated $\mathrm{Pt}^{3+}$ centers nor the $\mathrm{Fe}^{3+}-\mathrm{V}_{0}$ complex. The simple picture consistent with these observations is that the isolated Fe sites are changing their oxidation states, and are thus charge trapping centers as indicated below,

$$
\begin{array}{ll}
\text { optically written: } & \mathrm{Fe}^{4+}+\mathrm{e}^{-}-\cdots-\mathrm{Fe}^{3+} \\
\text { optically erased } & \mathrm{Fe}^{3+}+\mathrm{h}^{+} \text {-.-.- } \mathrm{Fe}^{4+}
\end{array}
$$

Figure 2: Plot of the remanent polarization, $\mathrm{P}_{\mathrm{r}}$, and isolated $\mathrm{Fe}^{3+}$ signal intensities for various UV-light/bias combinations. The written case was achieved by a $-6 \mathrm{~V} / \mathrm{UV}$ light treatment (propagation of electrons); the sample was erased by a $+25 \mathrm{~V} / \mathrm{UV}$-light combination (selective propagation of holes in the crystal).

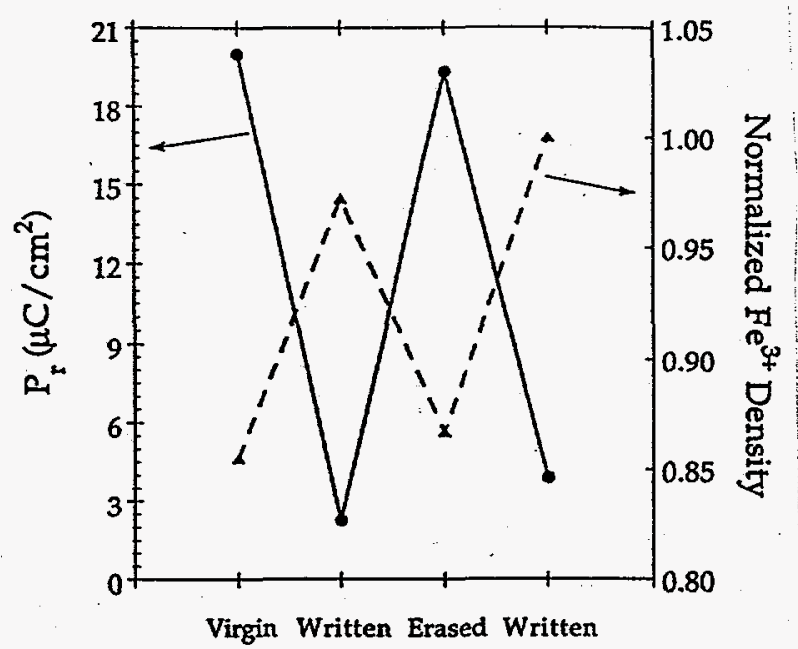

It should be stressed that eqns. (1) and (2) are only for the case in which the crystal is written with a negative bias and erased with a positive bias. If the BT crystal is written (subsequently erased) with a positive bias/UV (negative bias/UV) combination we find that the $\mathrm{Fe}^{3+}$ density decreases (increases) just as would be expected by the selective propagation of holes (electrons), thereby, demonstrating a symmetric response. Further supporting data for the interplay between $\mathrm{Fe}$ and the amount of switchable polarization can be found elsewhere [20].

Collectively, the EPR/P-V results spectroscopically support the notion that the suppressed polarization can arise by locking certain domain configurations by electronic charge trapping. 


\section{B. THERMALLY-INDUCED SUPPRESSION}

Next, we investigate the thermally-induced hysteretic changes in the BT crystal. Figure 3 shows the effects of various bias/thermal combinations. The suppressed P-V loop shown in Fig. 3 was taken after first poling the capacitor to negative remanence and then heating it to $80^{\circ} \mathrm{C}$ under a bias of $+5 \mathrm{~V}$. We find that the amount by which the P-V loop is suppressed strongly depends on the bias voltage, i.e., like the photo-induced effects of Fig. 1 (b), the temperature/bias suppression is also found to be maximized with a bias below the coercive voltage. A detailed study of the dependence on temperature has not performed.

This suppressed state can be optically restored by subjecting the films to UV-light while applying the saturating bias of polarity opposite to which the sample was originally subjected during the first heat treatment. Figure 3 illustrates the particular case in which the PZT film was optically rejuvenated by a UV/-50V combination at room temperature.

Figure 3: P-V measurements of $\mathrm{BaTiO}_{3}$ crystals subjected to various bias/thermal treatments: (a) initial, (b) $+5 \mathrm{~V} / 80^{\circ} \mathrm{C}$ combination for $60 \mathrm{~min}$., and (c) subsequent $-50 \mathrm{~V} / \mathrm{UV}$ process for $10 \mathrm{~min}$

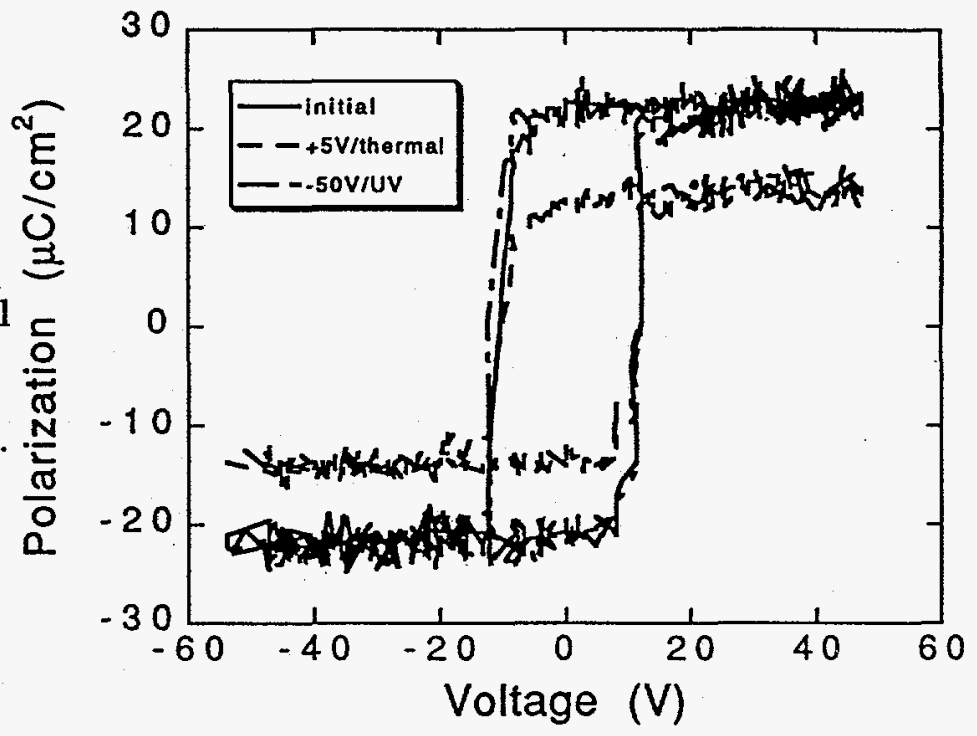

The observation that suppressed P-V loops can be optically restored via charge generation in the optical and thermal cases strongly implies a similar mechanism is responsible for locking the domains. As discussed earlier, we suggest that the mechanism involves the trapping of electronic charge at domain walls.

\section{ELECTRICALLY-INDUCED SUPPRESSION}

Figure 4 illustrates the P-E measurements of the BT crystal (a) before and (b) after electrical fatigue. As shown in Fig. 4, repeated polarization reversal has suppressed the amount of switchable polarization and has increased the coercive field markedly. The hysteresis loop in Fig. 4 (c) was taken after subjecting the fatigued sample with a $-100 \mathrm{~V} / \mathrm{UV}$ combination. This charge creation and subsequent propagation of electrons treatment only partially restores the suppressed remanent polarization by $\approx 5 \mu \mathrm{C} / \mathrm{cm}^{2}$. No further restoration was observed by a subsequent $+100 \mathrm{~V} / \mathrm{UV}$ combination. These results appear to indicate that the suppressed polarization state does not primarily arise by simply trapping electronic charge carriers at the domain walls. If this were the case, then charge carrier creation and migration via the 
bias/UV treatment should have completely restored the suppressed polarization as shown earlier [11,17-20].

EPR spectroscopy was used to investigate possible reasons for the degradation difference between fatigue and optically (thermally) induced polarization suppressions. We only report the salient features here; a greater discussion can be found elsewhere [22]. We find that fatigue decreases the isolated $\mathrm{Fe}^{3+}$ density and increases the $\mathrm{Pt}^{3+}$ density as shown in Fig. 5 . The decrease in $\mathrm{Fe}^{3+}$ comes about by either electron capture at $\mathrm{Fe}^{3+}$ forming diamagnetic $\mathrm{Fe}^{2+}$ ions, or hole capture at $\mathrm{Fe}^{3+}$ forming diamagnetic $\mathrm{Fe}^{4+}$ ions. The increase in $\mathrm{Pt}^{3+}$ signal likely arises by electron capture at $\mathrm{Pt}^{4+}$ sites. The fatigue-induced $\mathrm{Pt}^{3+}$ centers are stable at room temperature. We note that in the initial, un-fatigued $\mathrm{BT}$ crystals, the $\mathrm{Pt}^{3+}$ centers can only be metastably created by low temperature bandgap illumination $(\mathrm{T}<120 \mathrm{~K})$; these low temperature photo-induced $\mathrm{Pt}^{3+}$ centers anneal at $150 \mathrm{~K}$ [20]. Thus, when considering fatigue-induced and optically-induced $\mathrm{Pt}^{3+}$ stability differences, the simple fatigue-induced process involving electron capture to form paramagnetic $\mathrm{Pt}^{3+}$ centers is likely more complicated. It was suggested that a fatigue-induced distortion in the octahedron stabilized the trapped electron; this distortion may arise if an $\mathrm{V}_{0}$ migrates near the Pt ion [22].

Figure 4: P-E measurements for the $\mathrm{BaTiO}_{3}$ crystal (a) before and (b) after electrical fatigue. Loop (c) was taken after subjecting the fatigued crystal to a - 100V/UV combination. The sample was subjected to $4 \times 10^{8}$ cycles.

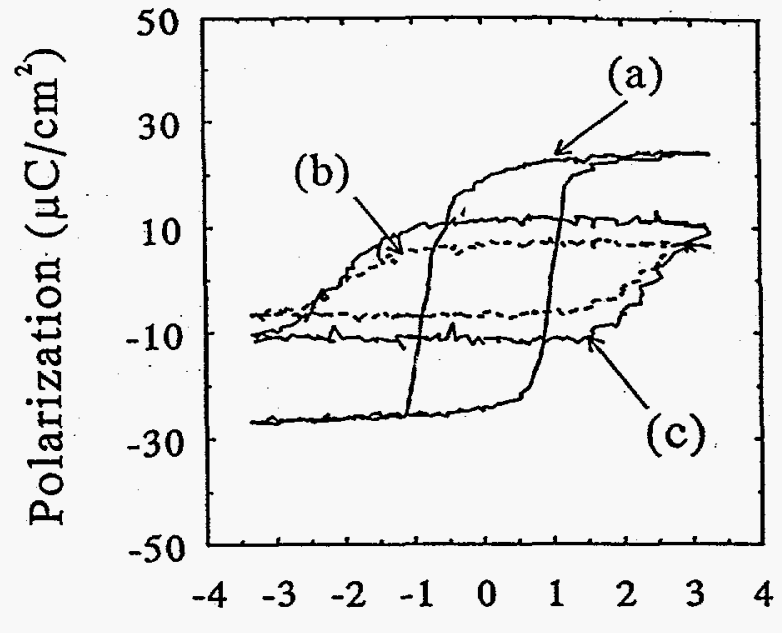

Electric field $(\mathrm{kV} / \mathrm{cm})$

Figure 5: Isolated $\mathrm{Fe}^{3+}$ and $\mathrm{Pt}^{3+}$ densities plotted as a function of switchable polarization during various stages of fatigue. The normalized $\mathrm{Fe}^{3+}$ density corresponds to $2 \times 10^{18} / \mathrm{cm}^{3}$ and the normalized $\mathrm{Pt}^{3+}$ density corresponds to $9 \times 10^{18} / \mathrm{cm}^{3}$.

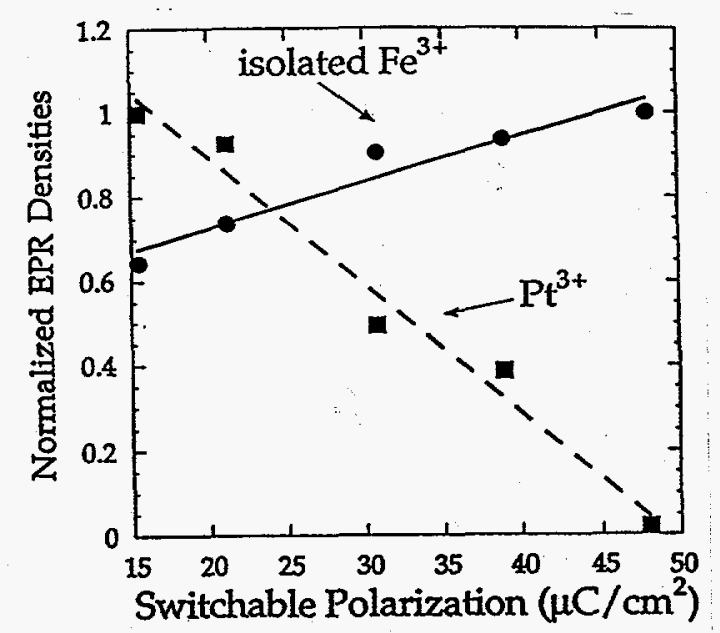


The notion that electrical fatigue can induce a local distortion in the octahedron was further supported by noting that the effective $g$ value $\left(g_{\perp}\right.$ eff), or the zero field splitting parameter, $D$ $[26,29]$, of the $\mathrm{Fe}^{3+}-\mathrm{V}_{0}$ complex changed with fatigue. $\mathrm{g}_{\perp}$ eff is determined by $\mathrm{D}$. As the fatigue process continues, the $\mathrm{Fe}^{3+} \mathrm{V}_{0}$ density decreases as does its $\mathrm{g}_{\perp}$ eff-value. Indeed, $\mathrm{D}$ is a sensitive function of the $\mathrm{Fe}^{3+}$ distance and position with respect to the $\mathrm{V}_{0}$ in perovskite materials [26]. In fact, $\mathrm{D}$ is determined by more than $70 \%$ by the next-neighbor ligand positions [28]. We interpret the changes in $g_{\perp}$ eff $(D)$ as experimental evidence that fatigue does induce distortions in the octahedron about $\mathrm{Fe}^{3+}$ centers. Furthermore, we find that changes in $g_{\perp}$ eff from the initial crystal values were only observed when the magnetic field is aligned along the fatigue electric field direction, no change was observed when the magnetic field is perpendicular to the electric field direction applied during fatigue. ( $\mathrm{g}_{\perp}$ eff is observed at $90^{\circ}$ to the [001] direction since rotation about the $(110)$ plane resulted in paramagnets whose $\mathrm{z}$ axes always remain perpendicular to the external magnetic field.) This suggests that local distortions are orientation dependent with respect to the applied electric field direction. The orientation-dependence in $\mathrm{g}_{\perp}$ eff is consistent with earlier observations by Pan, Yue and Tuttle [12], who found that in fatigued PZT ceramics that the initial polarization properties are measured when the direction of the applied field is changed $90^{\circ}$ with respect to the direction of the fatigue field.

From these results, Pan et al. [12] developed a fatigue model incorporating distortions of oxygen octahedra in the perovskite structure about an oxygen vacancy. The essence of the model is that the $\mathrm{B}$ cation ( $\mathrm{Ti}, \mathrm{Fe}, \mathrm{Pt}$ ) can become overdisplaced and locked into place due to a local potential well induced by a neighboring oxygen vacancy. Once locked into position, it can no longer participate in the overall polarization. To first order, our EPR results are consistent with this model inasmuch they provide direct experimental evidence for fatigueinduced distortions in oxygen octahedra about a B-site cation.

We note that the initial state of the BT crystals, with respect to the polarization value and EPR densities, could be rejuvenated by a $3 \mathrm{hr}$ reoxidizing anneal at $500^{\circ} \mathrm{C}$. To first order, this result suggests that oxygen vacancies may be involved in the degradation process induced by fatigue. The anneal either begins to fill up the oxygen vacancies, or causes their migration away from a domain wall.

Collectively, these results demonstrate three points. (1) They show that electrical fatigue may involve point defects as determined by changes in the oxidation state of various impurity-related defect centers. (2) The changes in oxidation state result from capture of electronic carriers and appear to be stabilized by induced local atomic distortions in the oxygen octahedran. (3) The distortions are orientation-dependent, occurring only along the electric field direction applied during fatigue.

D. CORRELATION BETWEEN OPTICAL, THERMAL, AND ELECTRICAL-INDUCED POLARIZATION SUPPRESSION IN BaTiO 3

At this juncture, we compare the fatigue results with those obtained for the optically and thermally induced polarization suppression. The optical and thermally-induced suppressed polarization was hypothesized to be due to trapping of electronic charge carriers at impurities 
(isolated Fe centers) near domain walls; the role of oxygen vacancies was not invoked. The photo-induced suppressed state was determined to be due to only electronic charge trapping at domain walls based on the following observations: (1) the photo-induced suppressed state could be rejuvenated by the creation and migration of charge carriers of the opposite sign to which it was originally subjected, (2) only isolated $\mathrm{Fe}^{3+}$ centers changed their oxidation states via charge trapping (no change in $\mathrm{Fe}^{3+}-\mathrm{V}_{0}$ or $\mathrm{Pt}^{3+}$ centers was observed, in contrast to that observed for fatigue), and (3) the thermal rejuvenation of the photo-induced suppressed polarization started to occur by annealing the crystal at $60^{\circ} \mathrm{C}$ for $15 \mathrm{~min}$.

In the fatigued BT crystals, the process is somewhat different: (1) domain reorientation did not occur to a large extent by the creation and migration of electronic charge in the crystal, (2) all three paramagnetic defects (isolated $\mathrm{Fe}^{3+}$ and $\mathrm{Pt}^{3+}$ centers and $\mathrm{Fe}^{3+}-\mathrm{V}_{0}$ complexes) changed their oxidation states, and (3) the thermal rejuvenation of the fatigue-induced suppressed polarization started to occur by annealing the crystal at $500^{\circ} \mathrm{C}$ for $15 \mathrm{~min}$. For the BT fatigue process, we suggest that electronic charge trapping is also likely involved; however, the degradation may also involve an additional feature that associates local oxygen octahedron distortions and oxygen vacancies in concert [22] to explain the differences between optical (thermal) and fatigue-induced polarization suppression. For fatigue, we suggest that the trapped charge carriers can become thermally and electrically stabilized by distortions in the oxygen octahedron, perhaps induced by nearby oxygen vacancies. It is assumed that the distortions make it difficult to restore the initial polarization by simply injecting electronic charge carriers into the crystal, or by a low temperature anneal. A greater discussion regarding further links between optically-; thermally-, and electrically-induced polarization suppression can be found in Section IV D.

\section{POLARIZATION SUPPRESSION IN PZT THIN FILMS}

\section{A. OPTICALLY-INDUCED SUPPRESSION}

We now discuss one of the photo-induced changes observed in PZT thin films. Figure 6 shows the P-V measurements of PZT thin films exposed to various UV/bias combinations. Figure 6 (a) shows the initial hysteresis loop of the film. The hysteresis loop shown in Fig. 6 (b) was taken after first poling the capacitor to $-\mathrm{P}_{\mathrm{r}}$ and then illuminating with bandgap light $(3.4 \mathrm{eV})$ under a bias of $+2 \mathrm{~V}$. The illuminated electrode is biased positively. with respect to the grounded bottom electrode. Just as observed for BT (Section III A), this UV/bias treatment has suppressed the switchable polarization This PZT thin film effect was first observed by Dimos et al. [17-19]. Their work showed that the amount by which the P-V loop is suppressed strongly depends on the bias voltage and wavelength of the UV light. Again, as observed for BT, the suppression is maximized by partially switching the thin films with a bias near the switching threshold (below the coercive voltage) and by illuminating with band gap light (hv $=3.4 \mathrm{eV}, \mathrm{Eg}$ $\approx 3.4 \mathrm{eV}$ ). The voltage dependence of the optically-induced polarization suppression for these films is shown in Fig. 7. In Fig. 7, suppression is only observed when positive biases are applied near the switching threshold with respect to $-\mathrm{P}_{\mathrm{r}}$, and when negative voltages are applied near the switching threshold with respect to $+\mathrm{P}_{\mathrm{r}}$. For a greater discussion of this effect please see Ref. [19]. 
Figure 6: P-V loops of PZT 40/60 films subjected to various UV/bias combinations: (a) initial, (b) $\mathrm{UV} /+2 \mathrm{~V}$ combination, and (c) UV/-15V combination. The UV/bias combinations were applied for $5 \mathrm{sec}$. The electrode material was Pt for the top and bottom electrodes.

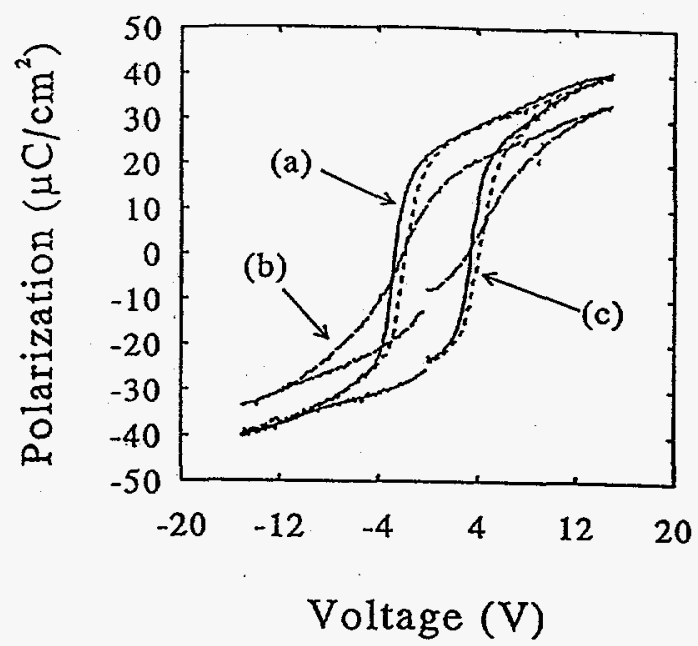

Figure 7: (a) Decrease in the amount of switchable polarization for PZT 40/60 films subjected to bias/UV combinations as a function of applied bias. The inset shows two of the corresponding P-V loops

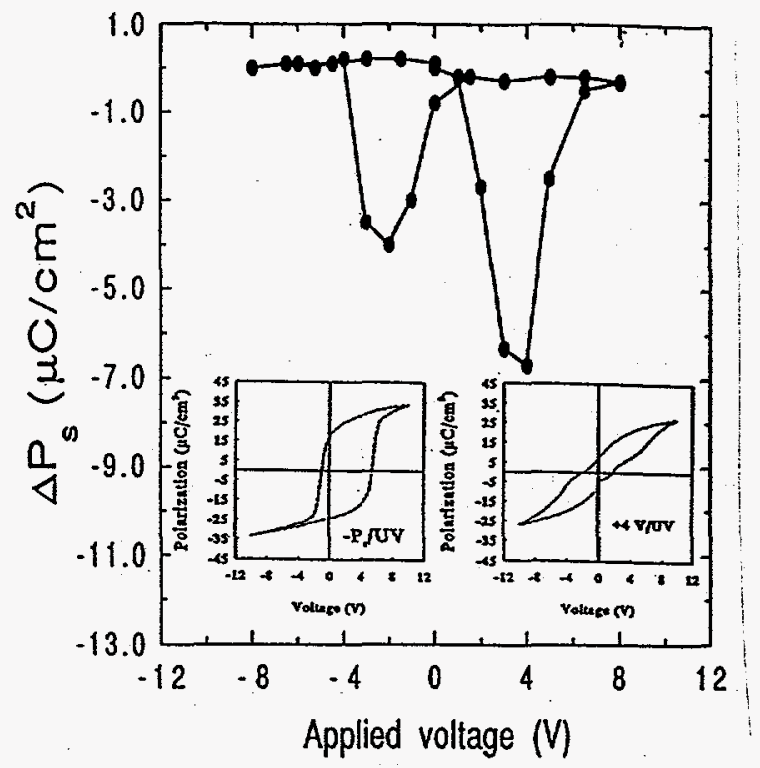

This written state, or suppressed polarization state, can be erased optically by subjecting the films to UV-light while applying a saturating bias of polarity opposite to which the sample was originally written. Figure 6 (c) shows the case in which the optically-written PZT film was erased by a UV/-15V combination. This optical restoration effect also causes a voltage shift in the P-V loop. The voltage shift effect is addressed elsewhere [17-19,30] and will not be discussed here. This effect likely arises since the light is creating electron/hole pairs throughout the film that can recombine with the trapped charge. We only mention at this time that these photo-induced effects are also observed in PZT films with $\mathrm{RuO}_{2}$ and LSCO electrodes. The effect is not electrode dependent to first order. 


\section{B. THERMALLY-INDUCED SUPPRESSION}

Next, we study thermally-induced hysteretic changes in PZT thin films. Figure 8 (a) shows the initial hysteresis loop of the film. The P-V loop shown in Fig. 8 (b) was acquired after first poling the capacitor to negative remanence and then heating the sample to $100^{\circ} \mathrm{C}$ under a bias of $+2 \mathrm{~V}$. This temperature/bias treatment has clearly suppressed both the remanent and switchable polarization. As illustrated in Fig. 9, we find that the amount by which the P-V loop is suppressed strongly depends on the bias voltage. Similar to the photo-induced effects of Fig. 7 , the temperature/bias suppression is also found to be maximized by partially switching the thin films with a bias below the coercive voltage; again, polarization suppression is only observed when positive biases are applied near the switching threshold with respect to $-\mathrm{P}_{\mathrm{r}}$ and when negative voltages are applied with respect to $+\mathrm{P}_{\mathrm{r}}$.

We find that this thermally-induced polarization suppression can be optically restored by illuminating the films with UV-light while applying the saturating bias of polarity opposite to which the sample was originally subjected during the first heat treatment. Figure 8 (c) illustrates the particular case in which the P-V response of this PZT film was largely restored by a $U V /-15 V$ combination at room temperature.

Figure 8: P-V loops of PZT 40/60

films subjected to various $100^{\circ} \mathrm{C} /$ bias combinations: (a) initial, (b) $+2 \mathrm{~V} / 100^{\circ} \mathrm{C}$ combination, and (c) $-15 \mathrm{~V} / 100^{\circ} \mathrm{C}$ combination. The P-V loops were taken at room temperature. The $100^{\circ} \mathrm{C} /$ bias combination was applied for $30 \mathrm{~min}$; the UV/bias combination was applied for $5 \mathrm{sec}$. The electrode material was Pt.

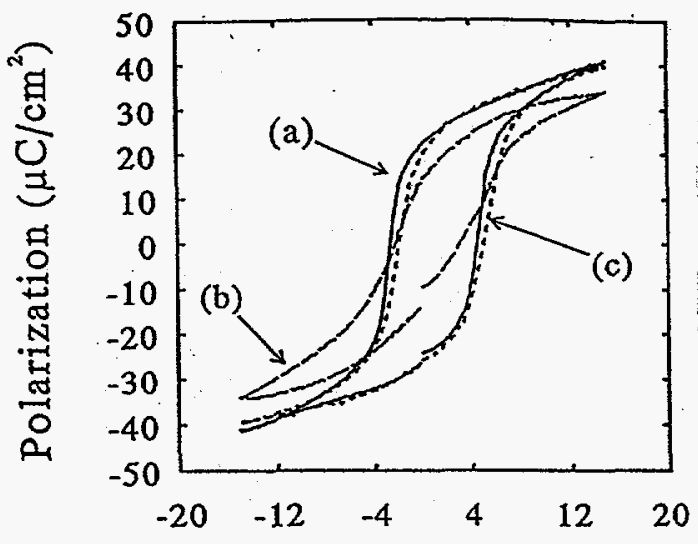

Figure 9: (a) Decrease in the amount of switchable polarization for PZT 40/60 films subjected to bias $/ 100^{\circ} \mathrm{C}$ combinations as a function of applied bias. The films were subjected to the bias/thermal combination for $15 \mathrm{~min}$ The inset shows two of the corresponding hysteresis loops taken at $100^{\circ} \mathrm{C}$.

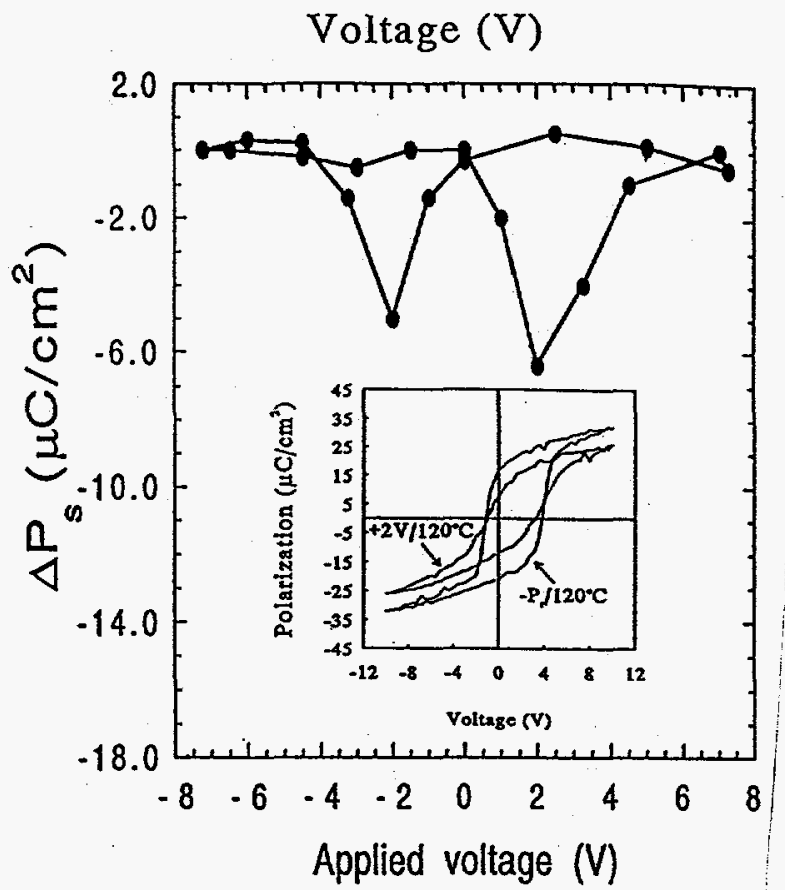




\section{ELECTRICALLY-INDUCED SUPPRESSION}

We now present our electrical fatigue results on Pt electroded PZT thin films. Figure 10 (a) shows the initial P-V loop of the film. The suppressed hysteresis loop shown in Fig. 10 (b) was taken after subjecting the capacitor to $3.5 \times 10^{8}$ voltage reversal cycles. Figure 10 (c) shows that this suppressed state can be optically restored to almost its initial state by subjecting the films to UV-light while applying a saturating bias. The rejuvenation of the P-V loop is, to first order, independent of the sign of the saturating voltage during the illumination. It should be stressed that optical illumination, or the applied saturating dc bias in and of itself, did not restore the suppressed polarization to any appreciable extent. It was a UV/saturating bias combination that led to the restoration of the suppressed polarization following fatigue, as also observed for the thermally and optically induced PZT effects. The optical restoration effects are not unique to the PZT 40/60 composition; restoration of the polarization was also observed in fatigued PZT films with $30 / 70$ and 53/47 compositions. Likewise, analogous fatigue/rejuvenation effects have been observed on PZT films deposited on $\mathrm{RuO}_{2}$ and $\mathrm{La}-\mathrm{Sr}-\mathrm{Co}-\mathrm{O}$ electrodes. These findings suggest that these effects are generic to PZT films.

Figure 10: PZT 40/60 Pt-electroded thin films subjected to various electrical and optical scenarios: (a) initial, (b) $3.5 \times 10^{8}$ cycles (electrical fatigue), and a

(c) UV/-15V combination.

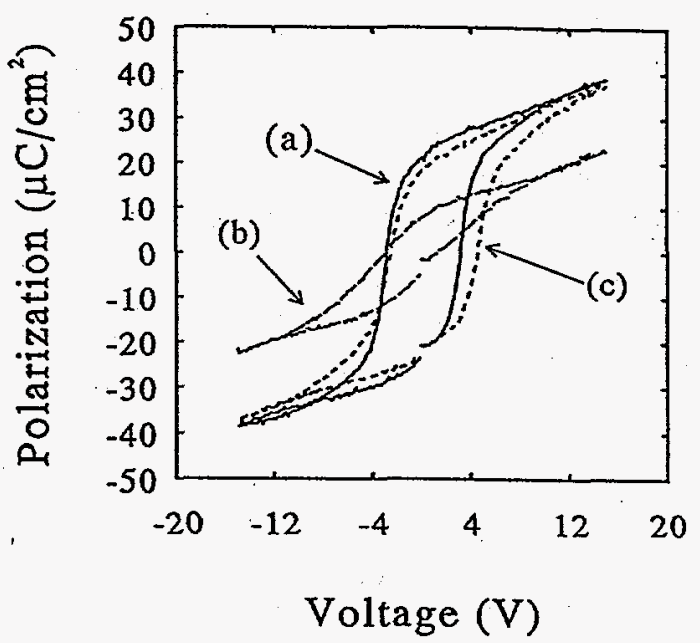

The fatigue-induced suppressed polarization is also largely restored by subjecting the ferroelectric capacitor to a saturation bias $/ 100^{\circ} \mathrm{C}$ combination $[11,31]$, This suggests that the UV-light and heat effects are linked. We believe the link is that they both produce mobile carriers in the ferroelectric material as discussed below.

\section{CORRELATION BETWEEN OPTICAL, THERMAL, AND ELECTRICAL-INDUCED POLARIZATION SUPPRESSION IN PZT THIN FILMS}

The observation that suppressed P-V loops can be optically restored via charge generation in all three PZT cases discussed thus far (optical, thermal, and electrical) strongly implies a similar mechanism is responsible for locking the domains. Just as proposed for the $\mathrm{BaTiO}_{3}$ crystals, we suggest that the suppressed polarization arises primarily by trapping electronic charge at domain walls. Some support for the electronic pinning mechanism may also be found by noting that the fatigue resistance improves when the capacitors are optically illuminated with UV light [15]. 
Spin resonance measurements were not performed on the PZT thin films; hence, the atomistic nature of the traps is not known.

Having suggested that the optical, thermal and electrical suppression effects in these PZT thin films are related to electronic charge trapping at domain walls, these results would then indicate that there is a source of mobile electrons and/or holes in all three cases. Obviously, the source of carriers is the electron/hole pair generation from the UV light in the optical scenario. As for the temperature/bias combination and the electrical cycling, free electrons and/or holes may come from a number of processes such as, carrier injection or emission over the metal/ferroelectric barrier.

These collective observations suggest that electrical fatigue in PZT thin films is predominantly due to electronic suppression of the polarization, and not from the migration and subsequent trapping of charged ionic defects such as oxygen vacancies or extended lattice defects pinning the domain walls $[3,7,13,16]$. Fatigue is induced by cyclic reversals of the saturating field. Fatigue with fields smaller than saturation typically results in less degradation [32]; thus, it appears that the high field is necessary to inject or create mobile carriers in the ferroelectric. For a fatigue mechanism, we propose that domain locking, due to the trapping of injected and/or created carriers, will occur primarily near the switching threshold field. Considering the optical and thermal results, we suggest that the switching threshold field is important in understanding the degradation caused by electrical fatigue. Recall, that both the optical [17-19] and thermal $[11,31]$ polarization suppression effects are only observed when a bias is applied near the switching threshold. This indicates that the ferroelectric needs to be biased in a favorable condition to trap the charge carriers. Bias fields near the switching threshold favor trapping because the ability to trap charge at misaligned domain boundaries is believed to be more likely.

At this juncture three points should be emphasized. First, an increase in the width of the P-V loop (measured by the difference in coercive voltages $-2 V_{c}$ ) is always observed following UVrejuvenation of the fatigued sample (Fig. 10 (c)). The observation of an increase in the width of the P-V loop following fatigue (without optical rejuvenation) has also been observed by Kwok and Desu [10]. An increase in the width of the P-V loop may occur if electrical fatigue induces a layer (hypothesized to be near the electrodes) that has a lower dielectric constant than the bulk of the film [8]. Hence, some of the applied voltage will be across this low dielectric constant layer, rather than being across the ferroelectric proper, making it appear as though the coercive voltage of the ferroelectric has increased. Consistent with this fatigue-induced surface layer, Scott et al. [33] have shown that an oxygen-deficient layer forms near the electrodes following fatigue in PZT capacitors. Second, following the optical rejuvenation of the fatigued capacitor, the rate of re-fatigue is more rapid [31]. This shows that even though the fatigue-induced suppressed polarization can be rejuvenated, the capacitor is not completely restored to its initial state. This may suggest that upon recombining with the opposite carrier type to free domain wall movement, that the defect cannot relax back to its original configuration; i.e., there may be some sort of structural relaxation that enables the defect to trap charge carriers more efficiently during the subsequent voltage cycling. This notion agrees with our $\mathrm{BaTiO}_{3}$ fatigue results (Section III C) discussed earlier, i.e., oxygen octahedron distortions. Third, even though most of the suppressed polarization can be restored by injecting electronic charge, a small fraction of the total remanent polarization $(\approx 10 \%)$ could not be rejuvenated. This small fraction may perhaps arise from a mechanism similar to that for the BT crystals, i.e., some of the trapped 
electronic charge is stabilized by distortions in the oxygen octahedron induced by oxygen vacancies. Indeed, the relative roles of electronic vs. ionic charge may change depending on bulk vs. thin film, doping, or oxygen partial pressure during sintering $[9,10,13,34]$.

\section{E. A SIMPLE FATIGUE TEST}

The correlation between electrical fatigue and the optically-induced suppression can be very useful. We find that films that can become optically suppressed also exhibit electrical fatigue; on the other hand, films that can not be optically suppressed also do not exhibit electrical fatigue as shown in Figs. 11 and 12, respectively. Generally speaking, the greater the extent of optical suppression, the greater the sample fatigues. This result strongly suggests that the bias/UV treatment can be used to test the fatigue properties of PZT thin films. This is a considerable time saving procedure. The bias/UV experiments typically take seconds to perform; whereas, fatigue measurements can take several days.

Figure 11: P-V loops of PZT films: (a) initial, (b) +3.0V/UV combination. The inset shows the corresponding fatigue characteristics of the films.

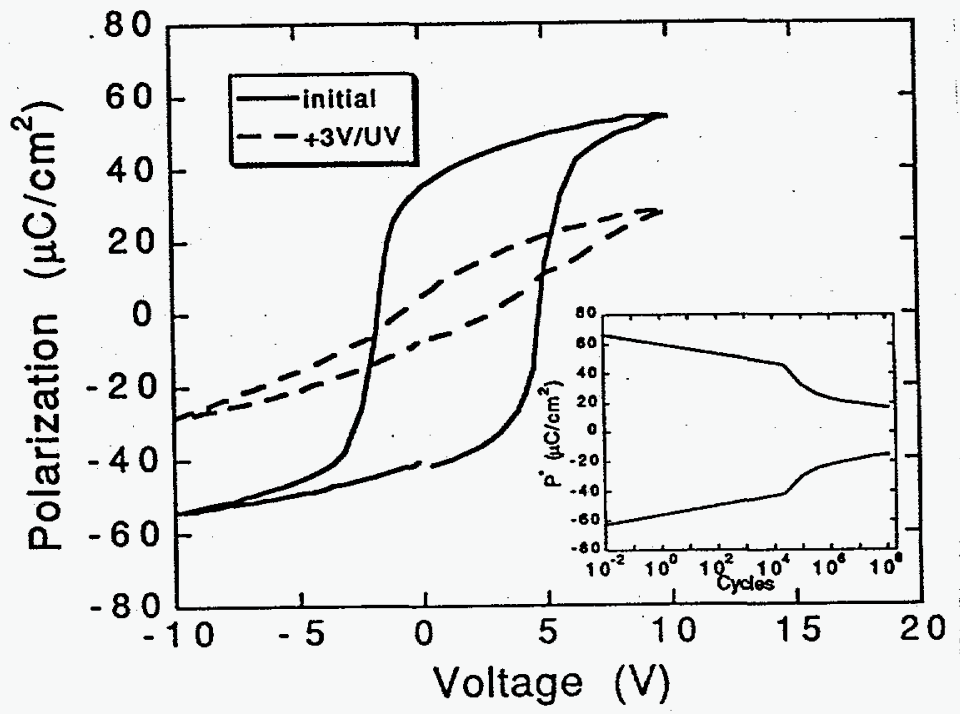

Figure 12: P-V loops of PZT films: (a) initial, (b) +0.6 V/UV combination. The inset shows the corresponding fatigue characteristics of the films.

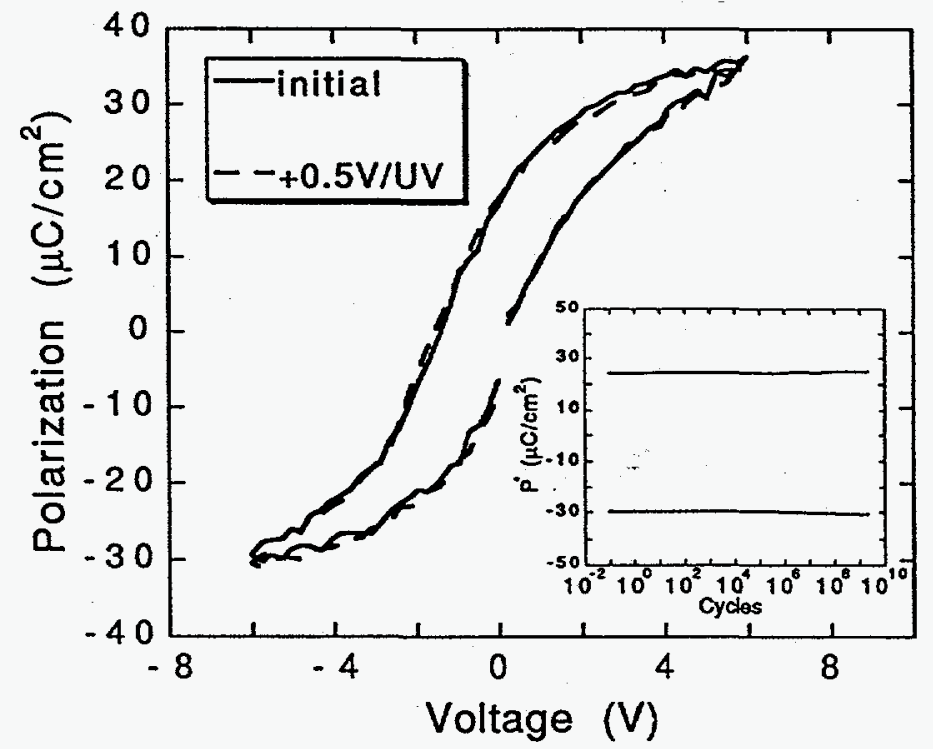




\section{CONCLUSIONS}

We have shown that electronic charge trapping, or charged ionic defects in concert with electronic charge trapping, can lock domain walls and lead to a suppressed polarization state in ferroelectric materials. In the $\mathrm{BaTiO}_{3}$ system, electronic domain pinning appears to predominate for the optically and thermally-induced polarization suppression. For electrical fatigue, we still suggest that electronic charge trapping is involved; however, the trapped charge can become thermally and electrically stabilized by fatigue-induced distortions in the oxygen octahedron. Nearby oxygen vacancies may be the stabilizing entity. The $\mathrm{BaTiO}_{3}$ fatigue results provide us with direct experimental evidence for local atomic distortions in oxygen octahedra induced by electrical fatigue in BT crystals. The distortions occur along the direction of the applied electric field during voltage cycling.

Electronic charge trapping appears to be the predominant mechanism for locking domains in sol-gel PZT films of various compositions, and electrode materials ( $\mathrm{Pt}, \mathrm{RuO}_{2}$, and $\left.\mathrm{LSCO}\right)$ when subjected to either electrical fatigue, a temperature/bias combination, or a UV/bias combination. For a PZT fatigue mechanism, we suggest that the high field during voltage cycling injects, or creates, mobile carriers in the ferroelectric. Once the carriers are injected into the ferroelectric, we hypothesize that the domain locking, due to the trapping of injected and/or created carriers, occurs primarily near the switching threshold field. Bias fields near the switching threshold favor trapping since misaligned domain boundaries, which can set up electrostatic potential wells to trap the charge, are viewed more likely.

Thus, there appears to be a strong link between optical, thermal, and electrical polarization suppression processes in PZT thin films. This being the case, we provide evidence that suggests that the bias/UV combination can be a very simple, straightforward, and quick test to determine the fatigue properties of PZT thin films.

\section{ACKNOWLEDGMENTS}

We are grateful to J.A. Alexander (ARPA) for many useful discussions during the course of this work. We would also like to thank Paul Grandon and Dale McIntyre for the $\mathrm{RuO}_{2}$ depositions. This work was supported by the U.S. Department of Energy under contract DE-AC0494AL85000 and by the Advanced Research Projects Agency through the National Center for Advanced Information Components Manufacturing.

\section{REFERENCES}

1. B.A. Tuttle, MRS Bull., 12, 40 (1987).

2. L.M. Shepard, Ceramic Bull., 71, 85 (1992).

3. J.F. Scott and C.A. Paz de Arajuo, Science, 246, 1400 (1989).

4. S. Dey and R. Zuleeg, Ferroelectrics, 108, 37 (1990).

5. R. Ramesh, W.K. Chan, B. Wilkens, H. Gilchrist, T. Sands, J.M. Tarascon, V.G. Keramidas, D.K. Fork, J. Lee, and A. Safari, Appl. Phys. Lett., 61, 1537 (1992).

6. J. Lee, L. Johnson, A. Safari, R. Ramesh, T. Sands, H. Gilchrist, and V.G. Keramidas, Appl. Phys. Lett., 63, 27 (1993).

7. I.K. Yoo, S.B. Desu, and J. Xing, MRS Symp. Proc., Vol., 310, 165 (1993)

8. P.K. Larsen, G.J. M. Dormans, D.J. Taylor, and P.J. van Veldhoven, J. Appl. Phys. 76, 2405 (1994). 
9. S.D. Bernstein, Y. Kisler, J. M. Wahl. S.E. Bernacki, and S.R. Collins, MRS Symp. Proc., Vol. 243, 373 (1992).

10. C.K. Kwok and S.B. Desu, MRS Symp. Proc., Vol. 243, 393 (1992).

11. W.L. Warren, D. Dimos, B.A. Tuttle, R.D. Nasby, and G.E. Pike, Appl. Phys. Lett., 65, 1018 (1994).

12. W.Y. Pan, C.F. Yue, and B.A. Tuttle, Ceram. Trans. 25, 385 (1992).

13. I.K. Yoo and S.B. Desu, MRS Symp. Proc., Vol. 243, 323 (1992).

14. C. J. Brennan, R. D. Parrella, and D.E. Larsen, Ferroelectrics, in press.

15. J. Lee, S. Esayan, A. Safari, and R. Ramesh, Appl. Phys. Lett., 65, 254 (1994).

16. Z. Wu and M. Sayer, submitted to Proc. Am. Ceram. Soc., PAC Rim Meeting, November, 1993, Honolulu, HI.

17. D. Dimos, W.L. Warren, and B.A. Tuttle, MRS Symp. Proc., Vol. 310, 87 (1993).

18. D. Dimos, W.L. Warren, M.B. Sinclair, B.A. Tuttle, and R.W. Schwartz, J. Appl. Phys., 76, 4305 (1994).

19. D. Dimos, B.G. Potter, M.B. Sinclair, B.A. Tuttle, and W.L. Warren, Integ. Ferroelectrics, 5, 47 (1994).

20. W.L. Warren and D. Dimos, Appl. Phys. Lett., 64, 866 (1994).

21. W.L. Warren, D. Dimos, B.A. Tuttle, and D.M. Smyth, J. Am. Ceram. Soc., 77, 2753 (1994).

22. W.L. Warren, B.A. Tuttle, and D. Dimos, Appl. Phys. Lett., submitted.

23. J.P. Remeika, J. Am. Chem. Soc., 76, 940 (1954).

24. C.E. Land and P.S. Peercy, Ferroelectrics, 22, 677 (1978).

25. E. Siegel and K.A. Muller, Phys. Rev. B20, 3587 (1979).

26. E. Siegel and K. A. Muller, Phys. Rev. B19, 109 (1979).

27. E. Possenride, P. Jacobs, and O.F. Schirmer, J. Phys. Conden. Matter, 4, 4719 (1992).

28. K. A. Muller, J. Physique, 42, 551 (1981).

29. W. Weltner, in "Magnetic Atoms and Molecules," (Dover, NY, 1983) p. 271.

30. G.E. Pike, W.L. Warren, D. Dimos, B.A. Tuttle, R. Ramesh, J. Lee, V.G. Keramidas, and J.T. Evans, Appl. Phys. Lett., in press (1995).

31. W.L. Warren, D. Dimos, B.A. Tuttle, G.E. Pike, P.G. Clews, and D.C. McIntyre, J. Appl. Phys. submitted.

32. W.H. Shepard, MRS Symp. Proc., Vol. 200, 277 (1990).

33. J.F. Scott, C.A. Araujo, B.M. Melnick, L.D. McMillan, and R. Zuleeg, J. Appl. Phys.,70, 382 (1991).

34. J.R. Anderson, G.W. Brady, W.J. Merz, and J.P. Remeika, J. Appl. Phys., 26, 1387 (1955).

\section{DISCLAIMER}

This report was prepared as an account of work sponsored by an agency of the United States Government. Neither the United States Government nor any agency thereof, nor any of their employees, makes any warranty, express or implied, or assumes any legal liability or responsibility for the accuracy, completeness, or usefulness of any information, apparatus, product, or process disclosed, or represents that its use would not infringe privately owned rights. Reference herein to any specific commercial product, process, or service by trade name, trademark, manufacturer, or otherwise does not necessarily constitute or imply its endorsement, recommanufacturer, or otherwise the United States Government or any agency thereof. The views and opinions of authors expressed herein do not necessarily state or reflect those of the United States Government or any agency thereof. 